\title{
Estabilidad de procesamiento de polímeros: índice de degradación en proceso
}

\author{
Andrés Felipe Rojas González ${ }^{1}$ y Laura María Aranzazu Ríos²
} 1Universidad Nacional de Colombia Sede Manizales, Departamento de Ingeniería Química, Campus la Nubia, Manizales, Colombia, anfrojasgo@unal.edu.co.
2Industrias Básicas de Caldas, km 18 vía Medellín, Manizales, Colombia, laura.aranzazu@qbasica.com.

\section{Resumen}

Se presentan los resultados del análisis de estabilidad en proceso para polietileno de alta densidad grado extrusión (PEAD-GE), polietileno de alta densidad grado inyección (PEAD-GI), polietileno de baja densidad (PEBD), poliácido láctico (PLA), policarbonato (PC), poliestireno (PS), polimetíl metacrilato (PMMA) y polipropileno (PP). Estos polímeros se reprocesaron en 5 ciclos de extrusión, inyección y extrusión/inyección. Cada polímero virgen y reprocesado se caracterizó respecto al índice de fluidez y se calculó su estabilidad en proceso mediante el índice de degradación en proceso (IDP). Se encontró que la estabilidad de los polímeros, de mayor a menor, durante la extrusión es: PEBD> PS> PMMA > PEAD-GI > PP> PLA, y en la inyección: PEAD-GI> PP> PLA >PC. Además, se determinó que la velocidad de giro del husillo no afecta la degradación en proceso.

Palabras clave: extrusión, inyección, IDP, reciclaje, reprocesamiento.

Editora: Pataquiva-Mateus, A. Y.

Citation: Andrés Felipe Rojas-Gonzálezy Laura María Aranzazu-Ríos, 2015. Estabilidad de procesamiento de polímeros: índice de degradación en proceso. Revista Mutis 5(1); pp. 37-45

Received: May 15, 2015; accepted: June 20, 2015; Published on line: June 30, 2015

Copyright: $\odot 2014$ Suárez et al. This is an open-access article, which permits unrestricted use, distributions and reproduction in any medium, provided the original author and source are credited.

Competing Interests: The authors have no conflict of interest.

\section{Stability of polymer processing: index of degradation in process}

\section{Abstract}

Results of stability analysis in process for High Density Polyethylene extrusion grade (HDPE-GE), High Density Polyethylene injection grade (HDPE-GI), Low Density Polyethylene (LDPE), Polylactic Acid (PLA),
Polycarbonate (PC), Polystyrene (PS), Polymethyl Methacrylate (PMMA) and Polypropylene (PP) are presented. These polymers are reprocessed in 5 cycles of extrusion, injection and extrusion/injection. Each virgin and reprocessed polymer was characterized respect to melt flow index and stability in process of each polymer by degradation index in process (IDP) was calculated. It was found that the polymers stability, from highest to lowest, during the extrusion is: LDPE $>$ PS $>$ PMMA $>$ HDPE-GI $>$ PP $>$ PLA and during the injection is: HDPE-GI> PP> PLA> PC. Furthermore, it was determined that the rotational speed of screw does not affect the degradation process.

Keywords: extrusión, inyección, IDP, recycling, reprocessing.

\section{Introducción}

En la actualidad los polímeros son utilizados principalmente en sectores como agricultura, comercio, in- 
dustria de automóviles y de empaques, en los que se aprovechan sus buenas propiedades mecánicas y su bajo costo (Ramis et al., 2004). Para su procesamiento industrial se emplean principalmente tecnologías como las de inyección y extrusión, y en menor proporción soplado, compresión y moldeo por transferencia. En cualquiera de estos procesos se obtienen recortes o sobrantes de polímero considerados residuos de producción, generalmente no biodegradables, los cuales conllevan a un aumento continuo de los desechos (Ramis et al., 2004). Sin embargo, estos residuos se pueden procesar nuevamente, con el fin de disminuir costos en materias primas y reducir el impacto ambiental (Goitisolo et al., 2008). El alimentar nuevamente el polímero al equipo, después de haber sido procesado, se le conoce como ciclo de procesamiento o reprocesamiento. En cada ciclo de procesamiento el material sufre degradación, la cual es irreversible debido a que experimenta cambios térmicos, mecánicos, físicos y químicos, que se reflejan en el detrimento en sus propiedades finales (Goitisolo et al., 2008). Estos cambios se deben principalmente a la exposición del material a la luz, a altas temperaturas, a la presencia de oxígeno (oxidación) y a esfuerzos mecánicos (Pandey et al., 2005).

Algunos estudios reportados en la literatura que analizan la degradación de polímeros sin procesar y reprocesados, lo hacen través de métodos de cambio de color, índice de fluidez, propiedades mecánicas y análisis termogravimétrico (Jančáŕ \& Tocháček, 2011; Hamester et al., 2015; Hermanová et al., 2009). Tocháček et al. (2011) y Hermanová et al. (2009) presentan la variación del índice de fluidez respecto al reprocesamiento de distintos polímeros. Otros investigadores han evaluado el comportamiento de diferentes propiedades mecánicas como resistencia a tracción, módulo de elasticidad, límite elástico, elongación y ruptura, además de la distribución del peso molecular en cada ciclo de procesamiento (Tocháček et al., 2008; Wang et al., 2012). Carrasco et al. (2010) emplearon el análisis termogravimétrico para el estudio de la degradación térmica de PLA. Igualmente, se ha establecido el índice de amarillez para el material procesado en varios ciclos como indicador de degradación (Tocháček et al., 2008; Tocháček et al., 2011; Hermanová et al., 2009). Tocháček et al. (2012) propusieron cuantificar la estabilidad térmica de polímeros que experimentan reprocesamiento, mediante el índice de degradación en proceso (IDP).
En este trabajo se busca establecer la estabilidad de procesamiento de 8 polímeros termoplásticos, al someterlos a 5 ciclos de reprocesamiento en extrusión, inyección y extrusión/inyección, mediante el cálculo del índice de degradación en proceso. Para ello se evalúa el efecto del tipo de proceso, tipo de polímero y velocidad de giro del husillo en extrusión sobre el IDP.

\section{Materiales y métodos}

\section{Caracterización del polímero virgen y reprocesado}

Los polímeros fueron suministrados por las empresas Nature Works e IDES Prospector - USA (en sus páginas de internet se pueden consultar las hojas de seguridad de los polímeros aquí estudiados). Para establecer la relación entre el tipo de polímero y su estabilidad térmica al experimentar reprocesamiento, se emplearon los polímeros: polietileno de alta densidad grado extrusión (PEAD-GE), polietileno de alta densidad grado inyección (PEAD-GI), polietileno de baja densidad (PEBD), poliácido láctico (PLA), policarbonato (PC), poliestireno (PS), polimetíl metacrilato (PMMA) y polipropileno (PP). Los polímeros vírgenes, llamados también polímeros sin procesar, se caracterizaron respecto a su temperatura de transición vítrea, fusión y cristalización por Calorimetría Diferencial de Barrido, en un calorímetro Netzsch DCS 200PC, según la ASTM D 3418-08. A estos polímeros y a los reprocesados, se les determinó el índice de fluidez (IF) mediante la norma ASTM D1238, en la que se establece la masa que fluye por un capilar en un tiempo de 10 minutos. Para ello se empleó un plastómetro marca Dynisco Polymer Test, modelo D4003.

\section{Procesamiento del polímero}

Los polímeros fueron reprocesados mediante los procesos de inyección, extrusión y extrusión/inyección. La inyección se realizó en una inyectora monohusillo marca Boy XS, con diámetro del husillo de $12 \mathrm{~mm}$, una relación L/D de 19.6, una velocidad máxima de inyección de $24 \mathrm{~cm}^{3} / \mathrm{s}$ y una presión máxima de 3130 bar (45.4 psi). La extrusión se llevó a cabo en una extrusora monohusillo marca EXTRUDEX modelo ED-N 45-30D, con un diámetro de husillo de $45 \mathrm{~mm}$, una relación L/D de 30 , y una restricción constante en la zona de dosificación de 10. Esta restricción varía entre 0 (máxima restricción) y 56 (mínima restricción). 
El sistema de calefacción de la extrusora está constituido por 8 zonas de calentamiento y la inyectora por 4 zonas de calentamiento. El proceso de extrusión/inyección consiste en pasar el polímero por la extrusora y después por la inyectora, tomando la muestra para análisis al final de cada inyección. Para establecer la relación entre el tipo de proceso y el IDP, los polímeros fueron reprocesados en 5 ciclos de inyección, 5 ciclos de extrusión y para el proceso de extrusión/inyección en 5 ciclos sucesivos solo se utilizó el PEAD-GI. Se tomaron 5 ciclos de reprocesamiento con el propósito de comparar resultados presentados en la literatura, dado que es común encontrar estudios para dicha cantidad de ciclos de reciclaje en proceso. Para determinar el efecto de la velocidad de giro del husillo en la extrusora sobre la estabilidad del polímero por el reprocesamiento, solamente se procesó PEAD grado $\mathrm{E}$ a 20 y $80 \mathrm{rpm}$; mientras que para determinar el efecto del tipo de polímero sobre el IDP, los polímeros fueron extruidos a una velocidad de giro de $30 \mathrm{rpm}$.

\section{Determinación del índice de degradación en proceso}

El índice de degradación en proceso (IDP) propuesto por Tocháček et al. (2012), es una forma cuantitativa de evaluar la estabilidad térmica de un polímero después de ser sometidos a múltiples reprocesamientos. La siguiente expresión es una modificación de la ecuación para el cálculo del IDP dada por Tocháček et al. (2012):

$$
I D P=A b s\left[\frac{\left(I F_{0}+I F_{1}+\cdots+I F_{\mathrm{n}}\right)-(n+1) I F_{0}}{(n+1) I F_{0}} \times 100\right]
$$

Donde $I F_{0}$ representa el índice de fluidez para el polímero virgen o polímero sin procesar, $I F_{1}$ a $I F_{n}$ son los índices de fluidez del polímero para los reprocesamientos 1 al $n$. Abs representa el valor absoluto de la ecuación sugerida por Tocháček et al. (2012). Este Abs corresponde a la modificación que se realizó a la ecuación, dado que se encontró que para algunos polímeros el índice de fluidez disminuye con el número de reprocesamientos, dando valores negativos para el IDP, lo cual se debe al tipo de degradación sufrida por estos (Mendes et al., 2011). Para el análisis de resultados, Tocháček et al. (2012) establecieron que un IDP de cero representa un polímero que no sufre degradación durante los reprocesamientos, mientras un IDP mayor que cero indica que el polímero se degrada por el procesamiento. Esto también es válido para la ecuación modificada, puesto que todo polímero experimenta degradación térmica con el reprocesamiento, pero algunos lo hacen mediante diferentes mecanismos de degradación (Cross linking, chain extension y branching) (Mendes et al., 2011).

\section{Razón de cambio de degradación en proceso}

La razón de cambio de degradación en procesos (RCD) se plantea para establecer qué polímero, entre los que se están evaluando, presenta una mayor degradación entre el primer y quinto ciclo de procesamiento, como se muestra en la siguiente expresión matemática:

$R C D=\frac{I D P_{5}-I D P_{1}}{I D P_{1}}$

Donde representa el IDP del polímero en su primer ciclo de procesamiento, y el es el IDP al experimentar 5 ciclos de procesamiento. Al comparar dos polímeros, el polímero que experimenta el mayor cambio de degradación entre el primer y quinto reprocesamiento, es aquel que tiene la mayor RCD; mientras que el polímero que experimenta mayor degradación debido al primer ciclo, reporta el menor valor de RCD.

\section{Resultados y discusión}

\section{Caracterización del polímero virgen y reprocesado}

Las características de temperatura de transición vítrea, fusión, cristalización, grado y estructura molecular del polímero se presentan en la tabla 1 para los 8 polímeros. En esta tabla se observa que el PC, PS y PMMA son polímeros amorfos (A), por lo tanto no reportan temperatura de cristalización, mientras que los otros materiales son semicristalinos (SC). EI PEAD, PC, PS y PP, son polímeros grado inyección (GI), el PEAD y PLA son grado extrusión (GE), mientras que el PEBD y el PMMA son grado extrusión/inyección (EI). También se aprecia que el PEAD grado I y el PC reportan el menor y mayor valor de la temperatura de transición vítrea, respectivamente. En cuanto a la temperatura de fusión, el PMMA tiene el menor valor y el PC el mayor valor de este parámetro. 
Tabla 1. Caracterización térmica de los polímeros por DSC

\begin{tabular}{|c|c|c|c|c|c|c|c|c|}
\hline Parámetro & PEAD & PEAD & PEBD & PLA & PC & PS & PMMA & PP \\
\hline $\begin{array}{l}\text { Temperatura de } \\
\text { transición vítrea, }{ }^{\circ} \mathrm{C}\end{array}$ & -113 & -111 & -105 & 63 & 145 & 98 & 100 & -25 \\
\hline $\begin{array}{l}\text { Temperatura de fusión o } \\
\text { ablandamiento, }{ }^{\circ} \mathrm{C}\end{array}$ & 129 & 131 & 112 & 210 & 220 & --- & 102 & 172 \\
\hline $\begin{array}{l}\text { Temperatura de } \\
\text { cristalización, }{ }^{\circ} \mathrm{C}\end{array}$ & 113 & 118 & 98.3 & --- & -- & --- & --- & 118 \\
\hline Grado del polímero & $\mathrm{Gl}$ & GE & IE & GE & $\mathrm{GI}$ & GI & EI & $\mathrm{Gl}$ \\
\hline Grado de cristalinidad & SC & SC & SC & SC & A & $A$ & A & SC \\
\hline
\end{tabular}

En las tablas 2 y 3 se presentan los valores de índice de fluidez para los polímeros procesados por extrusión y por inyección, respectivamente. Como se observa, el índice de fluidez para los polímeros PLA, PS, $P P$ y $P C$ se incrementa con el grado de reprocesamiento, este comportamiento se debe a la disminución en el peso molecular de los materiales en cada uno de ellos (Carrasco et al., 2010).

Por el contrario, el PEAD-GI, el PEBD y el PMMA presentan reducción en el índice de fluidez como consecuencia de la degradación por entrecruzamiento (cross linking), ramificación (branching) y extensión de la cadena principal (chain extension) durante cada ciclo de procesamiento (Rex et al., 2005). En la tabla 2 se observa que el índice de fluidez del PEAD-GE no se ve afectado por la velocidad de giro del husillo en la extrusora.

En la última columna de la tabla 3 se presentan los resultados del indice de fluidez para el PEAD grado inyección sometido al proceso combinado de extrusión/inyección. Aquí se observa que este parámetro disminuye con el reprocesamiento. Para el PC no fue posible determinar el indice de fluidez después del tercer ciclo de procesamiento, debido a que su fluidez supera el valor máximo que detecta el equipo.

Tabla 2. Índice de fluidez para los polímeros procesados por extrusión

\begin{tabular}{|c|c|c|c|c|c|c|c|c|}
\hline Ciclo & PEAD-GI & PEBD & PLA & PS & PMMA & PP & $\begin{array}{l}\text { PEAD-GE } \\
20 \mathrm{rpm}\end{array}$ & $\begin{array}{c}\text { PEAD-GE } \\
80 \mathrm{rpm}\end{array}$ \\
\hline 0 & 36.90 & 1.97 & 14.20 & 3.55 & 20.50 & 3.00 & 0.28 & 0.28 \\
\hline 1 & 34.30 & 1.82 & 17.10 & 3.85 & 18.40 & 3.08 & 0.36 & 0.36 \\
\hline 2 & 30.70 & 1.77 & 18.50 & 3.90 & 17.70 & 4.50 & 0.39 & 0.39 \\
\hline 3 & 25.90 & 1.73 & 21.50 & 3.95 & 16.80 & 4.10 & 0.42 & 0.43 \\
\hline 4 & 20.50 & 1.62 & 22.90 & 4.10 & 16.80 & 3.95 & 0.43 & 0.43 \\
\hline 5 & 18.60 & 1.50 & 24.70 & 4.60 & 16.50 & 4.70 & 0.42 & 0.42 \\
\hline
\end{tabular}

Tabla 3. Índice de fluidez para los polímeros procesados por inyección

\begin{tabular}{cccccc}
\hline Ciclo & PEAD-GI & PP & PLA & PC & PEAD-GI \\
\hline 0 & 36.9 & 3.0 & 14.2 & 48.5 & 36.9 \\
\hline 1 & 36.7 & 3.9 & 17.3 & 109.7 & 25.7 \\
\hline 2 & 33.4 & 4.1 & 23.1 & 331.4 & 24.2 \\
\hline 3 & 30.4 & 5.0 & 31.8 & - & 19.0 \\
\hline 4 & 28.4 & - & 39.7 & - & 14.5 \\
\hline 5 & 26.3 & - & 60.2 & - & 13.1 \\
\hline
\end{tabular}




\section{Efecto del tipo de proceso sobre el IDP}

El efecto del proceso de extrusión, inyección y extrusión/inyección, sobre el IDP para el PEAD-GI se presenta en la figura 1. Se observa que al analizar la degradación que ocurre entre un ciclo y el siguiente, la menor degradación experimentada por el polímero se da en el primer ciclo de procesamiento por inyección (IDP = 0.27), mientras que la mayor degradación ocurre en el proceso de extrusión/inyección, pero también en el primer ciclo (IDP = 15.18).

En los ciclos 1 a 5 el IDP varía aproximadamente en la misma proporción con una tendencia lineal para los 3 procesos que experimenta el polímero, confirmada por el coeficiente de correlación $\left(R^{2}\right)$ cercano a la unidad, con valores de 0.9972 para extrusión/ inyección, 0.9973 para extrusión y 0.9994 en inyección. Esto evidencia un efecto linealmente proporcional del reprocesamiento sobre la degradación del material. También se observa que el proceso de extrusión tiene un efecto de degradación del polímero aproximadamente 2 veces mayor en comparación con el proceso por inyección, esto confirma el hecho de que el proceso de inyección es menos agresivo que el proceso de extrusión.

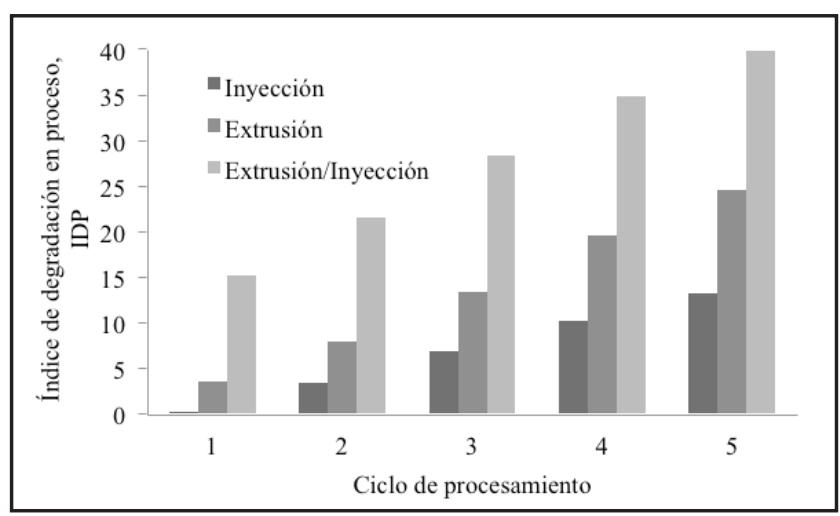

Figura 1. IDP para PEAD-GI procesado por extrusión, inyección y extrusión/inyección

En la figura 2 se compara el IDP para el PLA procesado por extrusión e inyección a través de 5 reprocesamientos. En esta figura se aprecia que el IDP para el PLA presenta la misma tendencia a lo observado para el PEAD-GI (ver figura 1) tanto para extrusión como para inyección. Sin embargo, al comparar la relación del efecto de los dos tipos de procesos, para el PLA se observa que a medida que el material se reprocesa por inyección, su degradación es mayor comparada con la extrusión, pasando de una degradación similar en los dos procesos para el primer ciclo de procesamiento, a ser tres veces mayor la degradación por inyección, en relación con la inyección, en el quinto ciclo de procesamiento.

Al comparar las figuras 1 y 2 , se aprecia que el IDP tiene valores de entre 0.2 y 40 para el PEAD-GI y entre 10 y 120 para el PLA. Esto confirma que el IDP depende del tipo de polímero, dado que está en función del $\mathrm{IF}$, que es característico de cada polímero. El efecto del tipo de polímero sobre IDP se analiza más adelante. Finalmente, se observa en las dos figuras que, sin importar el tipo de proceso, ambos polímeros sufren mayor degradación a medida que experimentan un mayor número de ciclos de procesamiento.

\section{Efecto de la velocidad de giro sobre el IDP}

En la figura 3 se presenta la variación del IDP, para el polímero PEAD-GE, con la velocidad de giro del husillo a 20 y $80 \mathrm{rpm}$ en el proceso de extrusión. En esta figura se observa que a las dos velocidades de giro, en el primer ciclo de procesamiento el polímero experimenta la máxima degradación (IDP de 14.82), esto comparado con la degradación observada entre un ciclo y el siguiente (como por ejemplo entre el ciclo 1 y 2 el IDP se incrementa aproximadamente 8 unidades). Igualmente se ve que la degradación en cada uno de los ciclos respecto al siguiente, disminuye a medida que aumenta el reprocesamiento.

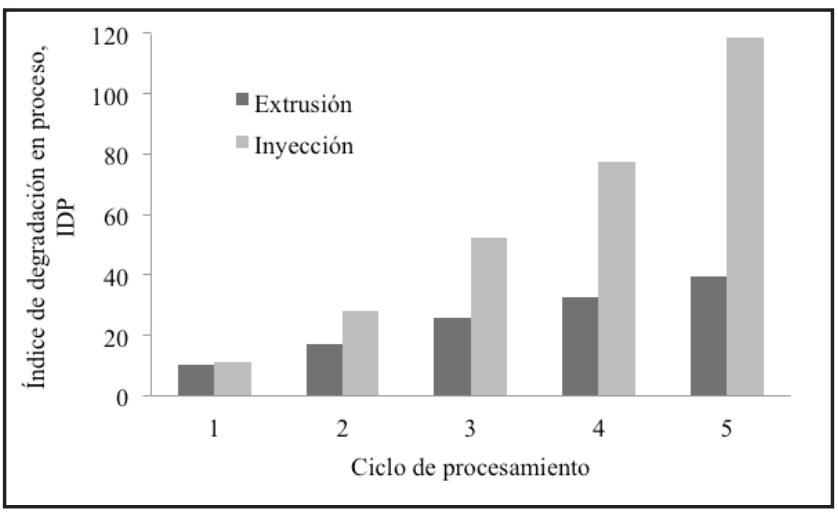

Figura 2. IDP para PLA procesado por extrusión e inyección

También se aprecia que al comparar el IDP a 20 y 80 rpm se obtienen valores similares de este parámetro en cada ciclo para ambos casos. Esto indica que la velocidad de rotación del husillo, en el proceso de extrusión, no afecta apreciablemente la degradación del 
polímero. Por lo tanto, los experimentos de extrusión se pueden llevar a cabo a cualquier velocidad de giro del husillo, entre estos dos valores, sin afectar los resultados de degradación del polímero. Es por ello, que a diferencia de estos dos experimentos, los demás ciclos de procesamiento por extrusión se realizaron a una velocidad de giro de $30 \mathrm{rpm}$.

\section{Efecto del grado del polímero sobre el IDP}

Al establecerse que la velocidad de giro del husillo en el proceso de extrusión no afecta considerablemente la degradación del polímero, se puede analizar el efecto del grado del polímero, ya sea grado inyección (figura 1) o grado extrusión (figura 3), sobre el IDP. Al comparar las figuras 1 y 3 , se evidencia que el PEADGI procesado por extrusión presenta un menor IDP para cada ciclo de procesamiento, comparado con el PEAD-GE; obteniéndose valores de degradación de entre 3 y 25 para el PEAD-GI, y de entre 14 y 38 para el PEAD-GE. Este comportamiento posiblemente se debe al mayor índice de fluidez del PEAD-GI, el cual permite que el polímero fluya sin requerir de un alto esfuerzo mecánico, como se espera que lo requiera el PEAD-GE.

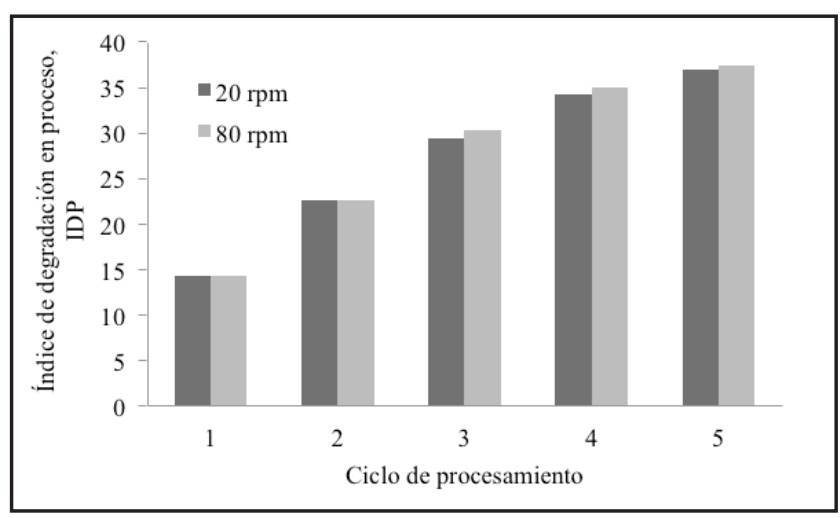

Figura 3. IDP para PEAD-GE procesado por extrusión a dos velocidades de giro de husillo

\section{Efecto del tipo de polímero en el IDP}

En las figuras 4 y 5 se muestran los resultados del cálculo del IDP para los polímeros procesados por extrusión (PEBD, PS, PMMA, PEAD-GI, PP y PLA) e inyección (PEAD-GI, PP, PLA y PC), respectivamente. Se puede ver en la figura 4 que en el primer reprocesamiento el PEBD sufrió su mayor degradación, luego descendió hasta el tercer ciclo y posteriormente aumentó levemente hasta el quinto ciclo, esto es al comparar la degradación experimentada por el polí- mero en un ciclo respecto al siguiente. Este comportamiento indica la existencia simultánea de dos mecanismos de degradación: el rompimiento de la cadena principal (chain scission) y el entrecruzamiento (cross linking) (Jin et al., 2012). Por lo tanto, se establece que durante los primeros procesamientos el rompimiento en la cadena principal domina sobre el mecanismo de entrecruzamiento. Sin embargo, a medida que aumentan los ciclos de proceso el mecanismo de entrecruzamiento prevalece.

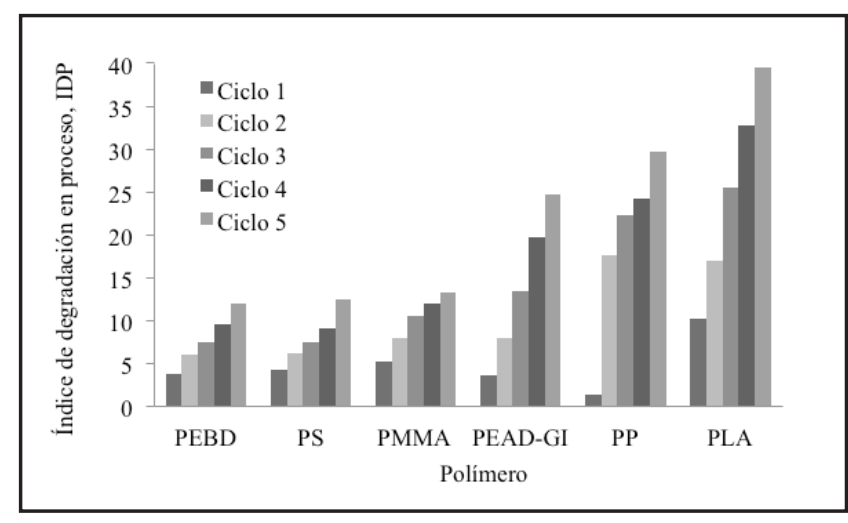

Figura 4. IDP para los polímeros procesados por extrusión

Al igual que el PEBD, el PS sufre su máxima degradación en el primer procesamiento como se observa en la figura 4. Para los ciclos siguientes, el aumento del IDP para el PS es menor que para el PLA y PEAD, esto refleja una mayor estabilidad térmica del PS en comparación con los otros materiales. El PS experimentó incremento en el índice de fluidez con los procesamientos sucesivos (tabla 2), lo cual se relaciona con una disminución en el peso molecular del material causado por mecanismos de rompimiento de la cadena principal (chain scission) (Vilplana et al., 2006).

Para el caso del PMMA, se observa una disminución progresiva de la degradación en proceso con cada ciclo de extrusión, siendo así el último reprocesamiento el de menor efecto, respecto a los ciclos anteriores. Lo anterior indica que el efecto degradativo en cada ciclo disminuye con el aumento del reprocesamiento, esto se debe a que el material se vuelve más refractario con el incremento de la temperatura (Badia et al., 2012).

Analizando el PP, se encuentra que su menor degradación la sufre durante el primer ciclo, lo cual indica que este ciclo no afecta considerablemente su estruc- 
tura molecular, conservando casi su estabilidad térmica como si fuera virgen. Sin embargo, el segundo ciclo degrada el material en una proporción de casi 8 veces la degradación sufrida en el primer ciclo; siendo esta la mayor degradación sufrida de un ciclo al siguiente por este polímero.

En la figura 4 se observa que, para todos los polímeros, la degradación aumenta con el número de ciclos de procesamiento por extrusión. Sin embargo, unos polímeros se ven más afectados que otros por el proceso de extrusión como es el caso del PP y PLA. Un orden de mayor a menor degradación por extrusión es: PLA > PP > PEAD-GI $>$ PMMA > PS >PEBD.

En la figura 5, para el PP se observa una mayor degradación en comparación con el material procesado por extrusión. Además, se aprecia que para el primer ciclo el IDP del material inyectado es aproximadamente el triple del material extruido (figura 4). Lo anterior posiblemente se debe a la mayor resistencia mecánica que presenta el material durante la inyección, por lo cual el polímero se ve forzado a tener mayor fluidez para ser inyectado. El incremento gradual del índice de fluidez con el reprocesamiento, demuestra que la degradación termomecánica sufrida por este polímero sigue el mecanismo de rompimiento de la cadena principal (chain scission) (Wang et al., 2012).

Para el PLA, se encontró que la degradación aumenta progresivamente del primero al último ciclo como se observa en la figura 5. Este incremento, el cual es debido al aumento en el índice de fluidez, refleja la disminución de la viscosidad y la reducción del peso molecular, provocado por la degradación del material en el procesamiento (Carrasco et al., 2010). También se aprecia que el IDP para este polímero, en los dos procesos (extrusión - figura 4, inyección - figura 5 ) y en cada uno de los reprocesamientos, es mayor en comparación con el IDP del PEAD-GI. Adicionalmente, a excepción del primer ciclo, el PLA presenta mayores valores del IDP respecto al PP inyectado y al PEAD-GI procesado por extrusión/inyección (figura 1). Lo anterior demuestra que el PLA experimenta una mayor degradación en proceso en comparación con otros polímeros. De igual manera se encontró que el incremento del IDP entre cada ciclo de inyección, es aproximadamente el triple que para el obtenido en el procesamiento por extrusión. Esto evidencia una mayor estabilidad en proceso del PLA al ser sometido a extrusión.

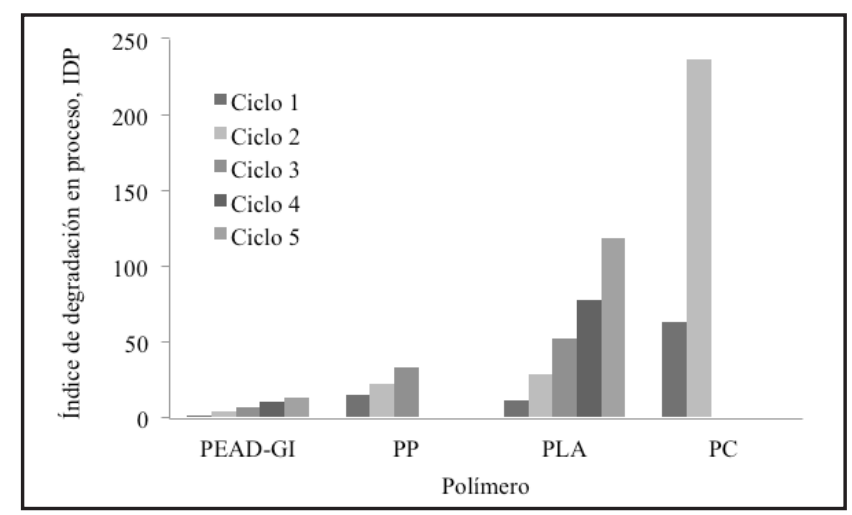

Figura 5. IDP para los polímeros procesados por inyección

EI PC únicamente fue procesado en dos ciclos, esto debido al gran incremento en su índice de fluidez en cada proceso. Como se observa en la figura 5, el IDP para $P C$ inyectado en el primer ciclo es mayor que el IDP para el último ciclo de PP y de PEAD-GI extruido e inyectado. Además, se encontró que el IDP del PC en el segundo ciclo es mayor que el IDP del quinto procesamiento del PLA, lo cual indica que el PC se degrada con mayor facilidad que los otros polímeros.

Por lo tanto, en la figura 5 se observa que para todos los polímeros la degradación aumenta con el número de ciclos de procesamiento. Sin embargo, unos polímeros se ven más afectados que otros por el proceso de inyección, como es el caso principalmente del PC, en el que después del segundo ciclo de procesamiento no fue posible medir el índice de fluidez, porque se encontraba por encima del límite superior posible de detección del equipo de análisis. Un orden de menor a mayor degradación por inyección es: PEAD-GI > PP $>$ PLA > PC, esto se explica porque los polímeros PLA y $P C$ son poliésteres que se hidrolizan y despolimerizan fácilmente debido a la presencia de humedad del ambiente, lo cual genera un aumento en la polidispersión que se refleja en un incremento drástico del índice de fluidez.

\section{Razón de cambio de degradación en proceso, RCD}

En la figura 6 se presentan los resultados de la razón de cambio de degradación entre el primer y quinto ciclo de procesamiento de los polímeros procesados en extrusión e inyección, dada por la ecuación (2). Como se observa en la figura 6, el PP y el PEAD-GI presentan la mayor razón de cambio de degradación entre los polímeros procesados por extrusión e inyección, respectivamente. 


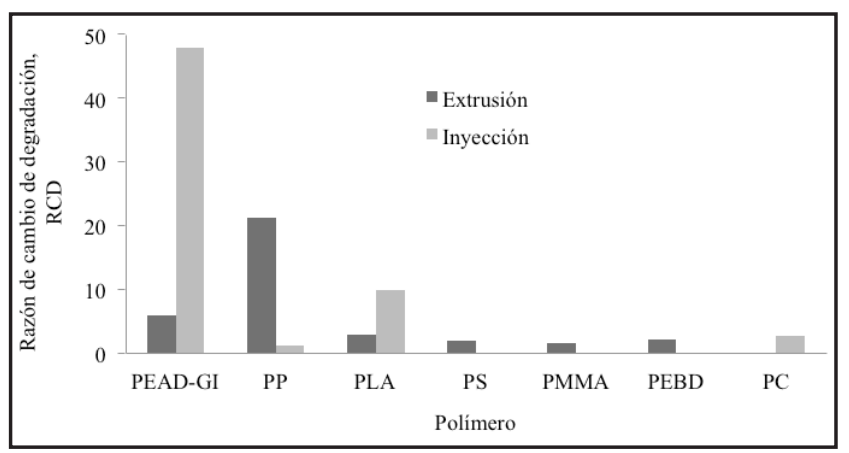

Figura 6. RCD para los polímeros procesados por extrusión e inyección

Este comportamiento refleja la influencia de los ciclos de reprocesamiento en la degradación del polímero. Para el PMMA, el PS y el PEBD procesados por extrusión se tiene un RCD bajo, esto quiere decir que la mayor influencia en la degradación del polímero hasta el ciclo 5, se presenta en el primer reprocesamiento. Para el PC se observa un RCD bajo, a pesar de tener los mayores valores de IDP de todos los polímeros procesados tanto por inyección como por extrusión. Esto muestra que la mayor influencia en la degradación del material se presenta en el primer ciclo de procesamiento. Para el PEAD-GI y el PLA se observa la mayor influencia en la degradación del polímero debido al ciclo 1 en el proceso de extrusión. A diferencia de estos, en el PP se presenta mayor influencia del ciclo 1 en la degradación del polímero durante el proceso de inyección.

\section{Conclusiones}

El orden de estabilidad térmica de mayor a menor para los polímeros procesados en cinco ciclos de extrusión es: PEBD>PS> PMMA> PEAD-GI> PP> PLA y para los polímeros procesados por inyección es: $P E A D-G I>P P>$ PLA >PC.

Polímeros como PP y PLA, que presentan mayor degradación térmica en procesos de inyección, son recomendados a ser reprocesamiento por extrusión.

Una velocidad de giro del husillo en la extrusora de entre 20 y 80 rpm no afecta la degradación en proceso del PEAD-GE. Se recomienda hacer lo mismo para otros polímeros y rango mayor de velocidad de giro, para establecer si esta conclusión se puede generalizar.

El PEAD-GI y el PMMA se degradan mediante mecanismos de entrecruzamiento, ramificación y extensión de la cadena principal. Por el contrario, el PLA, el PS, el PP y el PC se degradan por rompimiento en la cadena principal, mientras que el PEBD sufre degradación debido a mecanismos de rompimiento de cadena y de entrecruzamiento.

\section{Agradecimientos}

Los autores agradecen a la Universidad de Wisconsin (EE. UU.) por el apoyo en la obtención de las muestras en la extrusora e inyectora, y a la Universidad Nacional de Colombia por el apoyo económico para realizar la pasantía de investigación en la Universidad de Wisconsin.

\section{Referencias}

Badia, J. D., Santonja-Blasco, L., Martínez-Felipe, A., \& Ribes-Greus, A. (2012). Reprocessed polylactide: Studies of thermo-oxidative decomposition. Bioresource technology, 114, 622-628.

Carrasco, F., Pagès, P., Gámez-Pérez, J., Santana, O. O., \& Maspoch, M. L. (2010). Processing of poly (lactic acid): characterization of chemical structure, thermal stability and mechanical properties. Polymer Degradation and Stability, 95(2), 116-125.

Goitisolo, I., Eguiazabal, J. I., \& Nazabal, J. (2008). Effects of reprocessing on the structure and properties of polyamide 6 nanocomposites. Polymer Degradation and Stability, 93(10), 1747-1752.

Hamester, L. S., Muñoz, P. A. R., \& Canevarolo, S. V. (2015). A new device for in-line colorimetric quantification of polypropylene degradation under multiple extrusions. Polymer Testing, 41, 117-123.

Hermanová, S., Tocháček, J., Jančář, J., \& Kalfus, J. (2009). Effect of multiple extrusion on molecular structure of polypropylene impact copolymer. Polymer Degradation and Stability, 94(10), 17221727.

Jančář, J., \& Tocháček, J. (2011). Effect of thermal history on the mechanical properties of three polypropylene impact-copolymers. Polymer Degradation and Stability, 96(9), 1546-1556.

Jin, H., González-Gutiérrez, J., Oblak, P., Zupančič, B., \& Emri, I. (2012). The effect of extensive mecha- 
nical recycling on the properties of low density polyethylene. Polymer Degradation and Stability, 97(11), 2262-2272.

Mendes, A. A., Cunha, A. M., \& Bernardo, C. A. (2011). Study of the degradation mechanisms of polyethylene during reprocessing. Polymer Degradation and Stability, 96(6), 1125-1133.

Pandey, J. K., Reddy, K. R., Kumar, A. P., \& Singh, R. P. (2005). An overview on the degradability of polymer nanocomposites. Polymer degradation and stability, 88(2), 234-250.

Ramis, X., Cadenato, A., Salla, J. M., Morancho, J. M., Valles, A., Contat, L., \& Ribes, A. (2004). Thermal degradation of polypropylene/starch-based materials with enhanced biodegradability. Polymer degradation and stability, 86(3), 483-491.

Ramis, X., Cadenato, A., Salla, J. M., Morancho, J. M., Valles, A., Contat, L., \& Ribes, A. (2004). Thermal degradation of polypropylene/starch-based materials with enhanced biodegradability. Polymer degradation and stability, 86(3), 483-491.

Tocháček, J., Jančářr, J., Kalfus, J., Zbořilová, P., \& Buráň, Z. (2008). Degradation of polypropylene im- pact-copolymer during processing. Polymer Degradation and Stability, 93(4), 770-775.

Tocháček, J., Jančář, J., Kalfus, J., \& Hermanová, S. (2011). Processing stability of polypropylene impact-copolymer during multiple extrusion-Effect of polymerization technology. Polymer Degradation and Stability, 96(4), 491-498.

Tocháček, J., \& Jančář, J. (2012). Processing degradation index (PDI)-A quantitative measure of processing stability of polypropylene. Polymer Testing, 31(8), 1115-1120.

Vilaplana, F., Ribes-Greus, A., \& Karlsson, S. (2006). Degradation of recycled high-impact polystyrene. Simulation by reprocessing and thermo-oxidation. Polymer degradation and stability, 91(9), 2163-2170.

Wang, K., Addiego, F., Bahlouli, N., Ahzi, S., Rémond, Y., Toniazzo, V., \& Muller, R. (2012). Analysis of thermomechanical reprocessing effects on polypropylene/ethylene octene copolymer blends. Polymer Degradation and Stability, 97(8), 1475-1484. 